\title{
Performance of a Microbial Fuel Cell using a Magnet Attached Cathode
}

\author{
Changho Choi, Mia Kim, ${ }^{\dagger}$ Seok Won Hong, ${ }^{\ddagger}$ Yong Su Choi, ${ }^{\ddagger}$ Young II Song, ${ }^{\S}$ Sunghyun Kim, ${ }^{\#}$ and Hyung Joo Kim* \\ Department of Microbial Engineering, Konkuk University, Seoul 143-701, Korea. E-mail: hyungkim@konkuk.ac.kr \\ ${ }^{\dagger}$ Department of Microbiology, Pusan National University, Busan 609-735, Korea \\ ${ }^{\star}$ Water Environment Center, Korea Institute of Science and Technology, P.O. Box 131, Cheongryang, Seoul 130-650, Korea \\ ${ }^{\S}$ EIA division, Korea Environment Institute, Eunpyeong, Seoul 122-706, Korea \\ ${ }^{\#}$ Department of Bioscience and Biotechnology, Konkuk University, Seoul 143-701, Korea \\ Received February 2, 2010, Accepted March 26, 2010
}

Key Words: Membrane-less microbial fuel cell, Magnet, Cathode reaction

\begin{abstract}
Recently, a significant amount of attention has been focused on renewable energy sources that might help to satisfy increasing demand for energy consumption and environmental protection. From this perspective, microbial fuel cells (MFCs) are becoming an attractive prospect, owing primarily to their concurrent activities in wastewater treatment and electricity generation using wastewater as a fuel. ${ }^{1}$ An MFC is an electrochemical device which converts chemical energy to electrical energy using the catalytic activities of microorganisms. In recent years, significant effort has been expended toward the development of biological (i.e. bacteria and/or enzyme, etc.) and non-biological components (i.e. electrochemical mediator, electrodes, membrane, MFC structure etc.) for use in MFCs. Despite recent efforts to improve the electrical power performance of MFCs, there remain many technological barriers to overcome before the practical operation of a stable electrical power source becomes feasible. ${ }^{2}$
\end{abstract}

In the meanwhile, the membrane-less microbial fuel cell (MLMFC) using electrochemically active bacteria (EAB) is receiving increased attraction, due to its simplicity and the relative inexpensiveness of its components as compared to a conventional MFC system-for example, the MLMFC does not require an electrochemical mediator or an ion-selective membrane. ${ }^{3}$ One of the additional attractions of this type of arrangement is that it cannot merely generate electricity, but also consumes a broad range of practical organic wastes (e.g. animal or human sewage, organic wastewater from various fermentation processes, organic-rich sediment in freshwater and marine environments), owing to the comparatively simplicity of its construction. ${ }^{4}$ Because MLMFCs can utilize renewable substrates as feedstock, they also offer the prospect of long-term eco-friendly electrical energy generation. The approaches described above, however, have solved only some of the problems associated with conventional MFCs, and there remains a continuing need for a reliable electrical power source that will allow for more eco-friendly electricity generation. Attempts to increase the performance of MFCs have yielded several alternative methods based on electrochemical reactions, including membrane modification (e.g. membrane-electrode assembly), ${ }^{5}$ and electrode modification (e.g. Pt catalyst coating, rotating electrode, application of conductive polymer etc.). ${ }^{1,2,6,7}$

Previous studies have shown that a sufficient supply of oxy- gen, which functions as an oxidant for the cathode reaction, is one of the most crucial factors for the operation of MFCs. ${ }^{8}$ Gaseous oxygen is a unique gas that evidences paramagnetic properties due to its parallel spins in the electron configuration of a molecule. ${ }^{9}$ Due to this characteristic, the flow of oxygen gas can be controlled via magnetic force. It has recently been determined that this magnetic attractive force toward oxygen gas induces gas flow, and affects chemical reactions associated with oxygen gas-for example, combustion in diffusion flames and the cathode construction of a conventional chemical fuel cell. ${ }^{8,9,10}$

In this study, we evaluated the effects of a constant magnet on the cathode of an MLMFC, and also assessed the electrochemical performance of the magnet-attached MLMFCs. To the best of the authors' knowledge, this is the first report concerning the application of a constant magnet for the enhanced performance of a microbial fuel cell.

\section{Experimental Procedures}

MLMFC construction. Fig. 1 shows a schematic diagram of the membrane-less microbial fuel cell (MLMFC) employed in this study. ${ }^{3,5}$ The MLMFC consisted of an anode and cathode positioned at opposite sides of a poly-acrylic plastic cylindrical chamber $(\mathrm{d}=120 \mathrm{~mm}, \mathrm{~h}=200 \mathrm{~mm}$, empty bed volume of $c a$. $2000 \mathrm{~mL})$. Both the anode $(\mathrm{d}=80 \mathrm{~mm}, \mathrm{t}=18 \mathrm{~mm})$ and cathode $(\mathrm{d}=60 \mathrm{~mm}, \mathrm{t}=6 \mathrm{~mm})$ of the MLMFC were graphite felt (GF

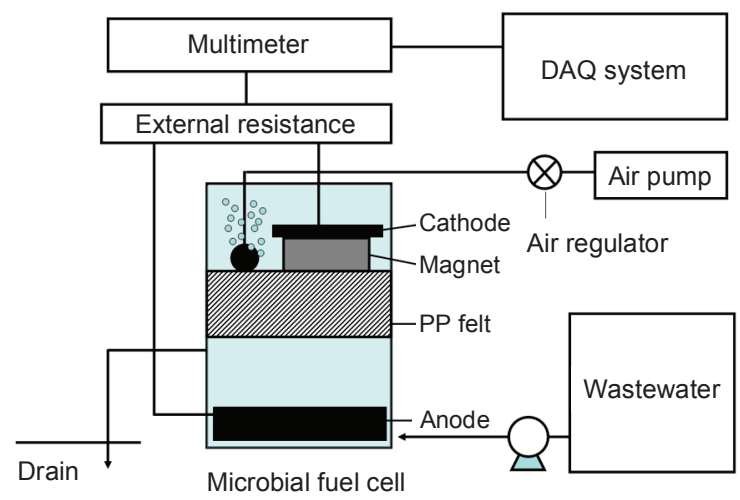

Figure 1. Schematic diagram of the MLMFC system used in the study. 
series, $6 \mathrm{~mm}$ in thickness, GEE Graphite Ltd, UK). The apparent surface electrode areas of the anode and cathode were 146 and $66 \mathrm{~cm}^{2}$, respectively. Polypropylene felt (MDK35W, $\mathrm{t}=70 \mathrm{~mm}$, Dongnam Felt Inc, Korea) was placed in the middle of the reactor to separate the electrode compartments. A block $(50 \times$ $50 \times 50 \mathrm{~mm}$ ) of constant magnet (Nd-Fe-B type, 0.64 Tesla, HK Inc, Korea) was placed at the underside of the cathode. The magnetic flux density of the magnet surface was determined with a Gauss meter (TM-601, Kanatec Co., Japan). The fuel was supplied to the bottom of the anode compartment and the effluent was removed via the drain port. The electrodes were connected with platinum wire at a resistance of $10 \Omega$ and a computer controlled data acquisition system (DAQ) was used to monitor and analyze the electricity from the MLMFC. In some experiments, the magnet and the aeration device were removed from the compartments.

Fuel, enrichment of EAB, and operation for the MLMFC. A modified artificial wastewater (containing $0.2 \mathrm{~g} / \mathrm{L}$ of dried milk, $0.15 \mathrm{~g} / \mathrm{L}$ of glucose, $\mathrm{pH}$ 7.0) was used as a fuel for the operation of the MLMFC throughout this study. For the enrichment of $\mathrm{EAB}$, the anode of the MLMFC was inoculated with activated sludge obtained from the Jungnang Sewage Treatment Plant (Seoul, Korea) and fed with the fuel in continuous mode with a flow rate of $0.2 \mathrm{~mL} / \mathrm{min}$ using a peristaltic pump. After inoculation and the a complete fuel fill-up of the system, the fuel supply rate was changed to $1 \mathrm{~mL} / \mathrm{min}$, and the current from the MLMFC was monitored using a digital multimeter (DAQ). ${ }^{5}$ Four sets of MLMFCs enriched for more than 3 weeks were used in this study. Whenever necessary, the cathode compartment was aerated at a rate of $200 \mathrm{~mL} / \mathrm{min}$. All experiments were conducted at least in duplicate, in a temperature-controlled chamber $\left(30{ }^{\circ} \mathrm{C}\right)$.

\section{Results and Discussion}

After inoculation and system operation using artificial wastewater as fuel, gradual increases in current were noted from all MLMFCs. The current increased gradually for 3 weeks, and achieved a steady value $\left(c a .2 .7 \mathrm{~mA} / \mathrm{m}^{2}\right)$ without aeration. In order to observe the effect of magnetic force on the performance, constant magnets were installed in the MLMFCs, and current generation under $10 \Omega$ was monitored (with and without aeration). As shown in Fig. 2, in the presence of aeration, rapid increases in current were noted. The largest increase in current was observed when the magnet was installed in the MLMFC in the presence of aeration $\left(\mathrm{ca} .22 .5 \mathrm{~mA} / \mathrm{m}^{2}\right)$. In the case of the MLMFC with no magnet in the presence of aeration, however, the current generation rate was relatively lower $\left(c a .19 .1 \mathrm{~mA} / \mathrm{m}^{2}\right)$ compared to that seen in the MLMFC featuring the magnet. In this experiment, the installation of the magnet induced an approximate $18 \%$ increase in current from the MLMFC.

When the experiments were conducted without aeration, the installation of the magnet into the MLMFC also induced an increase in current generation $\left(c a .4 .8 \mathrm{~mA} / \mathrm{m}^{2}\right)$ as compared to the control experiment $\left(2.7 \mathrm{~mA} / \mathrm{m}^{2}\right)$. According to these observations, it can be deduced that the installation of the magnet induces an increase in current generation, regardless of the presence or absence of aeration. Previous studies have shown

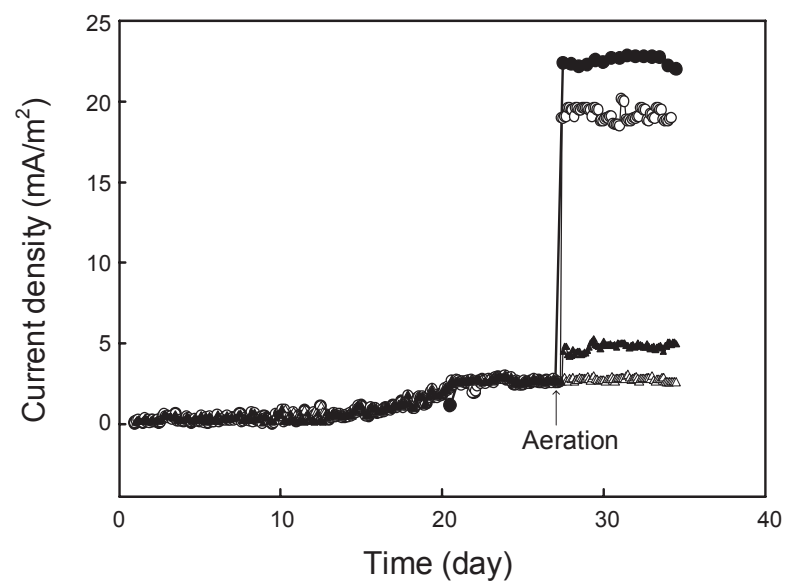

Figure 2. Effect of magnet on current generation from the MLMFCs with and without aeration. The arrow indicates the initiation of aeration $(200 \mathrm{~mL} / \mathrm{min})$ to the MLMFCs: with aeration with magnet (•), with aeration without magnet $(\mathrm{O})$, without aeration with magnet $(\mathbf{\Lambda})$, without aeration without magnet $(\triangle)$.

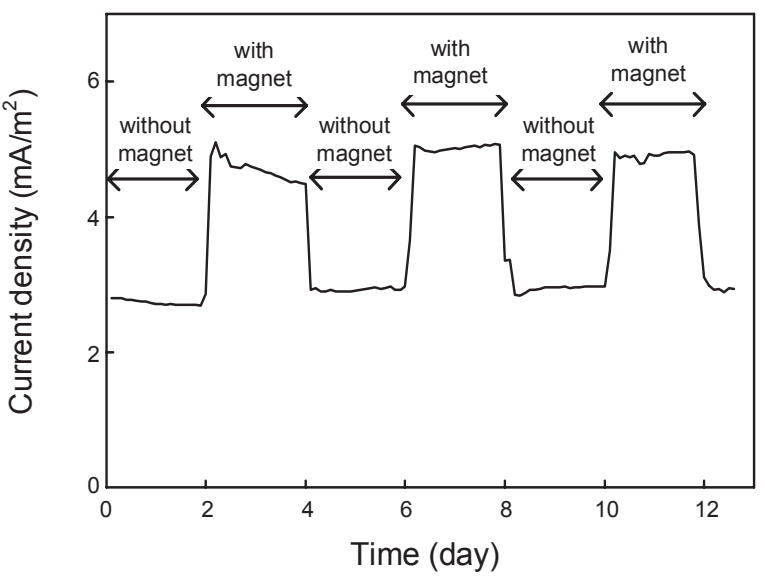

Figure 3. Current generation from the MLMFC system with and without the constant magnet. Current was measured with a resistance of $10 \Omega$.

that current generation from the MFC is proportional to the concentration of dissolved oxygen in the cathode compartment. ${ }^{3}$ However, the increase in the concentration of dissolved oxygen is limited by its solubility in water. ${ }^{8}$ Therefore, according to our experimental results, the attachment of a magnet could induce the increase in flux of the oxidant (i.e. oxygen) into the cathode surface. ${ }^{9,10}$

In order to clarify the effect of an installed magnet on current generation, a constant magnet was attached to the MLMFC that had been operated without aeration (Fig. 3). The experimental results clearly demonstrated that the presence of a magnet induced an increase in current from the MLMFC ( $c a$. from 2.7 to $4.9 \mathrm{~mA} / \mathrm{m}^{2}$ ). This experimental result also indicated, albeit indirectly, that the presence of a magnet (or magnetic field) near the cathode increases the oxidant concentration. It has been previously reported that one of the most salient problems in the operation of a conventional MFC is the energyconsuming forced aeration or addition of artificial oxidants, such as $\mathrm{K}_{3}\left[\mathrm{Fe}(\mathrm{CN})_{6}\right]$. In this experiment, it should be noted 

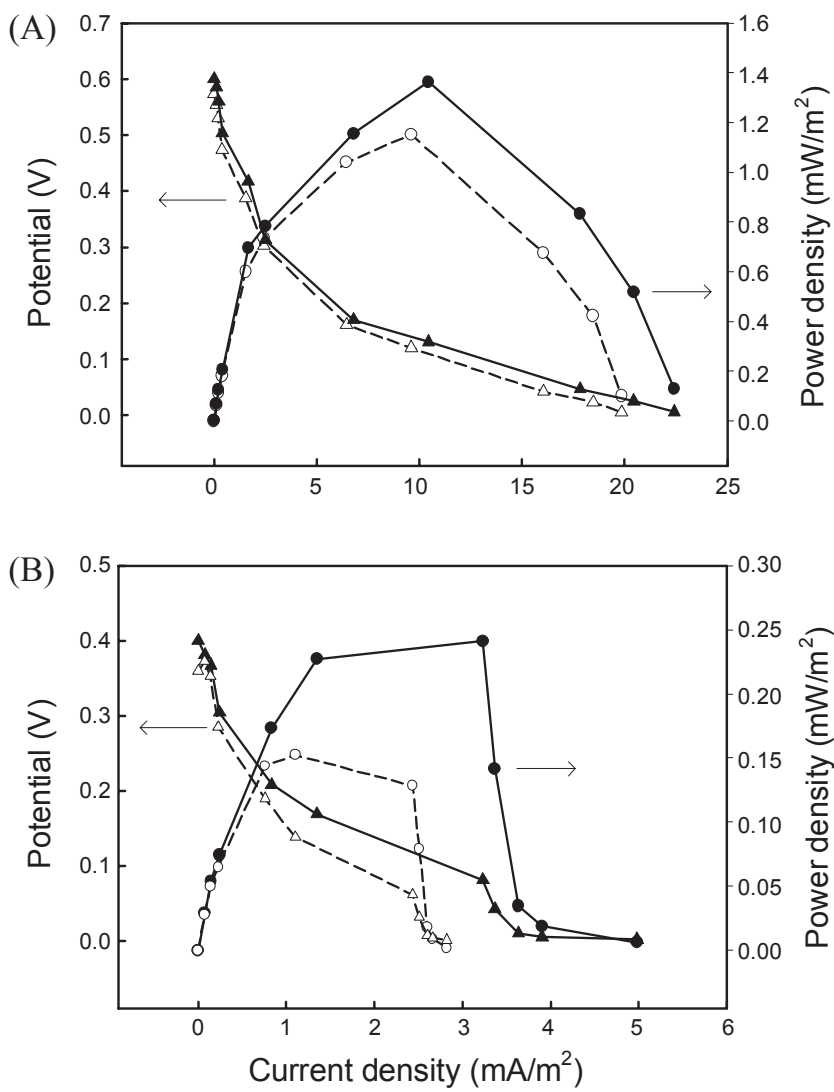

Figure 4. Comparison of MLMFC performance: polarization curves of MLMFC with aeration (A) and without aeration (B). The dotted lines show the experiment without the magnet.

that the installation of the magnet to the MLMFC induced an $81 \%$ increase in current as compared to that recorded in the MLMFC without an installed magnet.

Fig. 4 shows the effect of the installed magnet on potential and power density plots $v s$. current, with and without aeration. With aeration, the installation of the magnet on the MLMFC cathode caused an increase in power density of approximately $18 \%$ (from 1.15 to $1.36 \mathrm{~mW} / \mathrm{m}^{2}$ ). Without aeration, an increase in power density of about $73 \%$ was detected (from 0.15 to 0.25 $\mathrm{mW} / \mathrm{m}^{2}$ ). With the increase in current density (i.e. reduction in external resistance), the potential reduced pattern in the aerated MLMFCs was almost identical. However, in the case of MLMFC without aeration, the potential decreased more sharply in the
MFC without the installed magnet. A possible reason for the rapid decrease in potential from the MFC without both magnet and aeration could be the limitation of oxygen supply to the surface of cathode. These results suggested that the installation of the magnet increased the concentration of oxygen around the cathode as compared to the cathode without the magnet, thereby decreasing the mass transfer loss. ${ }^{8}$

In this study, we developed a new method to increase the electrochemical performance of MLMFC by applying magnets to the cathodes. The most troublesome issue in the construction of an MFC is the continuous supply of oxidants, such as oxygen, by energy-consuming devices. Therefore, the application of magnetic force to the cathode might prove to be a simple and inexpensive method to enhance the performance of MFCs. This method might be expected to be particularly beneficial in cases in which a sufficient amount of oxygen in the cathode area cannot be provided by artificial aeration (i.e. sediment MFCs). Further studies will be required to establish the electrochemical characteristics of MLMFCs under various experimental conditions, including the application of magnetic force to an anode compartment to remove the oxygen from the fuel.

Acknowledgments. This subject was supported by the Korean Ministry of Environment (The Eco-Technopia 21 project, No. 2008-08002-0075-0, 2008).

\section{References}

1. Davis, F.; Higson, S. P. J. Biosens. Bioelectron. 2007, 22, 1224.

2. Bullena, R. A.; Arnotb, T. C.; Lakemanc, J. B.; Walsha, F. C. Biosens. Bioelectron. 2006, 21, 2015.

3. Jang, J. K.; Pham, T. H.; Chang, I. S.; Kang, K. H.; Moon, H.; Cho, K. S.; Kim, B. H. Process Biochem. 2004, 39, 1007.

4. Hong, S. W.; Kim, H. J.; Choi, Y. S.; Chung, T. H. Bull. Korean Chem. Soc. 2008, 29, 2189.

5. Kim, M.; Hyun, M. S.; Gadd, G. M.; Kim, G. T.; Lee, S. J.; Kim, H. J. Environ. Technol. 2009, 30, 329.

6. Hea, Z.; Shaob, H.; Angenenta, L. T. Biosens. Bioelectron. 2007, 22, 3252 .

7. Kim, S.; Yuan, Y. Bull. Korean Chem. Soc. 2008, 29, 168.

8. Rismani-Yazdi, H.; Carver, S. M.; Christy, A. D.; Tuovinen, O. H. J. Power Sources 2008, 180, 683.

9. Okada, T.; Wakayama, N. I.; Wang, L.; Shingu, H.; Okano, J. I.; Ozawa, T. Electrochim. Acta 2003, 48, 531.

10. Wang, L. B.; Wakayama, N. I.; Okada, T. Chem. Eng. Sci. 2005, 60,4453 . 